\title{
CORRESPONDENCE
}

\section{Tungus Black Hole?}

SIR,-The interesting suggestion of Jackson and Ryan that the Tungus event was caused by a black hole (Nature, 245, $88 ; 1973)$ has a flaw. If the postulated interstellar population of small black holes inhabits the Galaxy, their mass density is $\gtrsim 10^{6} M_{\odot} \mathrm{pc}^{-3}$ (assuming a flux of 1 per century over the Earth), which is impossible. A cosmological habitat is equally impossible. Implausible alternatives are: (1) Those objects are confined to the solar system (why? gathered somehow during formation?); their total mass is $\gtrsim 10^{-2} M_{\circ}$. (2) These objects are much rarer, and the Earth is most fortunate to have received a visit in the last century. (3) One can somehow make as large a bang with a much smaller black hole.

Yours faithfully,

\section{Douglas M. EardLey}

W. K. Kellogg Radiation

Laboratory,

California Institute of Technology,

Pasadena,

California 91109

\section{Tungus Event}

SIR,--In regards to an objection raised by $\mathrm{Mr}$ Eardley to the black hole explanation of the Tungus event, I should point out that we do not make a claim for there being a flux of 1 per century over the Earth. In fact the only estimate of substellar mass black hole mass density is that of Hawking and others that it may be on the order of the cosmological mass defect.

A more serious question is why the object would have a velocity characteristic of the solar system. Can this be due to a complex perturbative encounter of the small mass with the Earth-Moon system? Would this explain the strange discrepancy in the longitude of the Moon for the period of 1900 to $1920^{1}$ ?

$$
\text { Yours faithfully, }
$$

$$
\text { A. A. JACKSON }
$$

Department of Physics,

The University of Texas at Austin, Austin,

Texas 78712

${ }^{1}$ Munk, W. H., and MacDonald, G. J. F., The Rotation of the Earth (Cambridge University Press, 1960).

\section{Transcription Unit}

SIR,-During the proceedings of the Thirteenth International Congress of Genetics in Berkeley (August 20-29, 1973) it became apparent that there is now a definite need to have operational terms for the units of transcription and translation, particularly in eukaryotic systems. I suggest the terms 'transcon' and 'translon' for the units of transcription and translation respectively. A transcon-as presently understood by the work of people like Richard Firtel of the Massachusetts Institute of Technology, Cambridge, and Brian McCarthy of the University of California, Santa Barbara-includes that segment of the total DNA sequence on a chromosome which is located between two spacer sequences; a spacer sequence consists of about twenty-five thymine-adenine base pairs (poly(A) in mRNA). A transcon has a total length of about 30,000 base pairs in insects and has alternating sets of repetitive and unique sequences of DNA. A transcon thus has several structural genes (unique sequences) and is obviously different from a replicon in eukaryotes or an operon in prokaryotes. A translon may be defined as the unit of translation at mRNA level. It may be as small as a single gene, for example in the case of haemoglobin mRNA, or as large as a hundred or so genes, for example, perhaps, in the case of histone mRNA.

\section{Yours faithfully, SURESH C. Goel}

Department of Zoology,

University of Poona,

Poona 7

\section{Reprints for USSR}

SiR,-I I have just today received a request from Professor E. M. Nadgornyi of the Institute for Solid State Physics of the Academy of Sciences of the USSR in Moscow for a reprint of one of my scientific articles as well as for exact details of special search techniques for digital computer simulation.

While requests of this sort are not infrequent, in this instance I hesitated to send the information requested because of the recent increased repression of Soviet advocates of civil rights and of Soviet scientists who have applied for permission to emigrate to Israel.
Because of the immediate dismissal of leading Soviet Jewish scientists and engineers from the professional positions and their virtual scientific ostracism following such visa applications, I decided to adopt a different approach in sending the requested information in this particular instance.

I have written to Professor E. M. Nadgornyi and informed him that while I personally favour cooperation, collaboration, and dissemination of information between scientists of all countries, I cannot condone the official attitudes of the Soviet government in respect of such noted scientists as Professors Lerner, Levich, and Sakharov. I am, therefore, sending all of the information requested by Professor Nadgornyi regarding special computer simulation techniques to Professor Aleksander Lerner, a noted cyberneticist and computer scientist. I am informing Professor Nadgornyi that he can obtain these documents by contacting Professor Lerner, a fellow Muscovite.

I feel that this may be a very important method by which scientists and engineers in the western world can make it clear to their counterparts in the USSR that such repression of Soviet Jewish scientists and, indeed, of many Soviet civil rights activists such as Sakharov and Solzhenitsyn is counter to any East-West detente, be it political, cultural, or scientific. I therefore urge others to follow my example in this matter of transmitting reprints and other information to the USSR.

\section{Yours faithfully, Marc Herbert Richman}

Division of Engineering,

Brown University,

Providence,

Rhode Island 02912

\section{Diving Terns}

SIR,--Dr Dunn's observations on the diving success of terns ${ }^{1}$ are very interesting, but $I$ think do not take account of what may be the most important fish behaviour contributing to their surviving the unwelcome attentions of such birds of prey. I refer to the "Mauthner Reflex", the startle-response of teleost fish, which is discussed in detail elsewhere ${ }^{2}$. A bird in free fall from a height of $10 \mathrm{~m}$ would, after hitting the water, 